EOMmUn: Communication et organisation

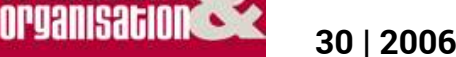

Modèles et modélisations, pour quels usages ?

\title{
De l'utilité des recherches-actions en SIC
}

\section{Vincent Meyer}

\section{OpenEdition}

Journals

Édition électronique

URL : http://journals.openedition.org/communicationorganisation/3455

DOI : 10.4000/communicationorganisation.3455

ISSN : 1775-3546

\section{Éditeur}

Presses universitaires de Bordeaux

\section{Édition imprimée}

Date de publication : 1 octobre 2006

Pagination : 89-108

ISSN : 1168-5549

\section{Référence électronique}

Vincent Meyer, " De l'utilité des recherches-actions en SIC », Communication et organisation [En ligne], 30 | 2006, mis en ligne le 21 juin 2012, consulté le 19 avril 2019. URL : http://journals.openedition.org/ communicationorganisation/3455 ; DOI : 10.4000/communicationorganisation.3455

Ce document a été généré automatiquement le 19 avril 2019

(c) Presses universitaires de Bordeaux 


\title{
De l'utilité des recherches-actions en SIC
}

\author{
Vincent Meyer
}

1 Comme le montre bien la thématique du $\mathrm{XV}^{\mathrm{e}}$ congrès de la Société française des sciences de l'information et de la communication (Questionner les pratiques d'information et de communication. Agir professionnel et agir social, Bordeaux, 2006), les recherches doivent être en prise avec les préoccupations actuelles des exercices professionnels et se situer dans une coproduction de savoir entre des experts et des profanes ayant un rapport différent à la situation ou à un objet technique à vocation communicationnelle. Les sciences de l'information et de la communication (SIC) demeurent « un espace scientifique et social [avec] ses zones de contact et ses interfaces avec les autres disciplines d'une part, et avec la société d'autre part» (Boure, 2002: 9); en tant que telles, elles ne peuvent rester coupées des réflexions sur les finalités d'une praxis. Reste à faire fructifier le bénéfice que peut retirer la recherche en information et communication des interrelations avec des professionnels pour qui il reste possible de faire vivre une pratique, sans en référer au regard ou au discours scientifique ; en situation d'expertise au quotidien, ils en «savent long » même s'ils ne savent pas l'objectiver.

2 Dans cet article, on souhaite rappeler que la recherche-action (RA) reste une démarche adaptée pour développer des stratégies permettant de soutenir des professionnels attachés au développement de leur métier. En effet, faire reconnaître la recherche endogène qu'ils mènent commande de les soutenir dans leur manière de construire l'objet et les aider dans la découverte des travaux théoriques sur leurs organisations, missions et technologies. L'enjeu est de taille pour le développement des formations (permanentes et continues) à la recherche. Par ailleurs, il faut reconnaître que la participation des professionnels facilite aussi l'actualisation des savoirs théoriques. On développera peu ici les questions relatives au rapport entre la connaissance et l'action, qui s'enlisent toujours à un moment ou un autre dans le problème du statut et de la légitimité des savoirs des uns et des autres, montrant qu'il existe «quelque part » une hiérarchisation des savoirs ou une « vraie » culture.

3 L'expression recherche-action est maintenant bien connue; ses différents principes, déclinaisons et techniques d'enquête font toutefois l'objet d'emprunts divers et 
d'utilisations variées. Elle est souvent conçue comme un outil de travail permettant de faire participer à l'analyse savante et/ou à l'évaluation de leurs résultats les membres d'une organisation donnée. Elle reste un dispositif essentiel pour développer des synergies entre la recherche académique et la recherche appliquée liée à une demande sociale; les interventions expertes des chercheurs et les connaissances produites dans l'acte par des praticiens de terrain.

Pour objectiver certaines des conditions d'objectivation dans ce type de démarche préoccupation épistémologique chère à Pierre Bourdieu (1980) - et engager le débat sur les RA, le propos sera centré autour de trois questions :

- Comment un partage de savoir permet-il de rendre compte de transformations dans des pratiques d'information et de communication?

- Que signifie «saisir les points de vue des individus et faire qu'ils deviennent les savants de leur propre réalité »?

- Quel statut accordons-nous au savoir « profane » en dehors de sa valeur sociale?

\section{Les productions de connaissances opérationnalisables : le chercheur agent du changement?}

5 Si l'on suit Rémi Hess (1983: 12), la paternité du concept de recherche-action est généralement attribuée à Kurt Lewin (1890-1947). Dans la recherche-action lewinienne «à certains moments, le chercheur se transforme en formateur et en agent de changement. Il intervient directement auprès des populations pour modifier leur comportement [...]. La démarche de Lewin peut se caractériser comme la recherche des moyens les plus efficaces de conduire un changement social désiré ». L'étude très popularisée de Kurt Lewin, pour les autorités américaines, sur les comportements alimentaires au début des années 40 , reste un bon point de départ pour comprendre - audelà des fondements de la dynamique des groupes (autre paternité que peut réclamer ce psychologue allemand) - la nécessité de développer des recherches actions sur les conditions essentielles de la réception des informations. Finalement la double question que se pose Kurt Lewin est : comment incorporer différemment les énergies mobilisées pour se conformer, construire des connaissances opérationnalisables facilement diffusables?

6 Quelques détails s'imposent pour rappeler, à la suite de Gilles Amado et André Guittet (1997), ce grand classique de la recherche. Dès 1942, la pénurie, due à la constitution de réserves pour l'armée américaine, conduisit les autorités à se pencher sur le moyen de changer les habitudes alimentaires des Américains. Ainsi fallait-il convaincre le plus grand nombre de la valeur nutritive de certains bas morceaux de viande. Les résultats obtenus par différentes communications orientées ou persuasives: campagnes d'information par presse, par radio, par affichage, s'étaient révélés minimes. Celles-ci avaient surtout tenté de démontrer les avantages économiques de ce changement. Kurt Lewin décida de porter son action sur de petits groupes de ménagères, actrices principales dans la décision d'achat de la viande. Il a montré que, même dans des groupes restreints, la diffusion d'informations expertes, qualifiées et/ou spécialisées, démontrant le pourquoi et le comment consommer, a eu un impact négligeable. En l'espèce, apporter de l'information - surtout individuellement -, c'était augmenter la pression vers un 
changement. En revanche, interpeller et solliciter des tiers intéressés pour qu'une solution aboutisse, laisser la discussion se développer sans intervenir i.e. limiter l'expertise permettaient des modifications significatives d'attitudes en réduisant les résistances au changement. Autrement dit, le fait de parler et de s'impliquer dans une discussion de groupe, qui remet en cause une habitude sociale, participerait à réduire les craintes.

7 En fait, l'apport principal de Kurt Lewin porte sur les modes d'action possibles pour faire accepter des informations (persuader de la valeur nutritive des bas morceaux de viande et montrer les avantages économiques d'une consommation généralement indicatrice d'un bas statut social) et modifier des jugements négatifs (réduire ou dépasser des craintes) versus des comportements (changer des habitudes alimentaires). Il a constaté que dans une population donnée certaines personnes jouent un rôle de facilitation du changement ce sont celles qui sont convaincues de son utilité. Sans pour autant être des leaders d'opinion, ce sont les personnes à convaincre en priorité. Car dans une population donnée, elles filtrent et/ou transmettent les informations, se comportent en «portiers ». Ellesjugent ou jaugent les informations acceptables pour le groupe et par leur action ou leur exemple, réduisent des appréhensions ou des tensions. Ainsi chaque groupe possèdet-il son champ dynamique avec ses canaux de communication, ses frontières, ses barrières, ses portiers. Une information nouvelle est mieux acceptée dans la mesure où elle s'intègre dans un processus de construction collective d'une représentation, qui passe notamment par une étape de mise en commun des perceptions, de confrontation, de négociation et de délibération entre différentes subjectivités.

8 La modélisation lewinienne peut encore s'appliquer à de nombreux champs professionnels. Ces travaux montrent surtout l'intérêt pour le chercheur, de ne pas simplement recueillir des données auprès de différents acteurs et/ou de se "réserver l'analyse", mais de les associer à ces étapes. C'est ici qu'on peut aussi replacer la problématique interactionniste $\mathrm{du}$ "dedans", dans laquelle il est nécessaire pour les chercheurs de partager autant les tâches d'organisation des pratiques que celles liées à la démarche de recherche. De facto, les fondements épistémologiques de la RA s'ancrent dans la tradition ethnographique. Mais comme le souligne Georges Lapassade (1991), l'ethnographie est un regard porté sur la société (si on participe à sa vie c'est pour mieux la regarder), alors que dans la recherche-action, c'est l'action qui prime et pas le regard. Ainsi Patrick Boumard (1989:26/27) précise-t-il que : «L'analyse ne pourra se faire jour que du lieu même où se produit le sens. Autrement dit l'analyse sera interne ou ne sera pas".

\section{Accompagner les savants de l'intérieur}

9 Deux principes guident toute recherche-action et limitent la plurivocité de ses acceptions :

- L'idée selon laquelle toute action peut être source d'une « connaissance » immédiatement réinvestie dans l'activité en cours.

- L'absence de confrontation entre un savoir « savant » et des compétences profanes déjà là.

10 Certainement peut-on y voir un reliquat de la doctrine le playsienne associant l'observation des faits sociaux, la recherche et l'action et/ou un développement de la sociologie compréhensive d'inspiration wéberienne. Dans les deux principes, on 
reconnaît les membres d'un groupe comme les véritables savants de leur propre réalité (Boumard, 1989). D'où l'intérêt pour eux de se doter d'équipement de recueil des données et d'une légitimation ex post de ce savoir. Dans ce cas, il s'agit toujours de mettre en œuvre une démarche collective et participative et d'utiliser différents outils de production de connaissance. À n'en pas douter, les pratiques professionnelles constitueront de plus en plus le fondement même de l'intervention experte du chercheur et notamment en SIC. Ce faisant, on bouleverse les formes habituelles de l'investigation méthodologique. La demande de participation des chercheurs à la fabrication des activités de travail ou de mise en perspectives gagnera en importance. Pour ces derniers, développer les RA, c'est aussi accéder à des terrains variés, apprendre à négocier à la fois les conditions de l'activité de recherche et les contraintes d'engagement des participants, définir des techniques d'observation (périphérique, active, complète) et de recueil de données plus adaptées (e.g. des méthodologies projectives et participatives telles la méthode des scénarios ou les focus groupes). De la sorte, ces recherches permettront de saisir différemment les constructions subjectives en situation d'intervention.

Dans le cadre d'un développement de RA, l'expertise savante du chercheur est convoquée pour saisir et traduire, avec ses mots, le cadre et, en bonne logique, pour évaluer et donc labelliser l'action entreprise. Les questions de modes, d'opportunités ou de lois de séniorité font que certaines disciplines des sciences humaines et sociales sont plus représentées que d'autres, notamment la sociologie, la psychologie et les sciences de gestion. Ainsi, dans ce dernier domaine, propose-t-on des méthodologies aux bases épistémologiques solides, souvent centrées sur des actions participatives visant la totalité d'une organisation (entreprise, établissement, institution, etc.). En retour, cette discipline est l'une de celles qui transforment assez systématiquement l'expérience des terrains en savoirs actionnables (Avenier, 2004). Cette recherche opérationnelle (également appelée recherche-intervention en sciences de gestion ${ }^{1}$ ), fer de lance du management stratégique (identifier les sources de la connaissance dans l'organisation et les processus d'actions stratégiques) se retrouve dans les formes traditionnelles de la RA, qu'il nous faut promouvoir dans les SIC, dans une autre logique que celle des stratégies managériales, en particulier dans la gestion des ressources humaines.

Dans de nombreux champs d'application des pratiques d'information et de communication (e.g. l'intervention sociale, la médiation, la prévention des risques, le management), les praticiens sont à la recherche d'un discours garant de l'acte et dans une demande de connaissance immédiatement opérationnalisable. Les SIC peuvent donc sans difficulté trouver leur place dans pareille démarche, dans la mesure où nombre de leurs travaux traitent de :

- La clarification et l'approfondissement de la réflexion sur les chaînes décisionnelles, sur les principes qui président les décisions au nom de certaines valeurs (e.g. le bien commun, le juste et l'injuste, la solidarité...) ou savoirs.

- La conduite du changement et la gestion des crises internes dans un contexte d'évolution généralisée de dispositifs législatifs ou de démarches d'évaluation et/ou qualité, des plans et stratégies de communication.

- L'intellectualisation et l'objectivation des activités de travail qui reposent pour beaucoup sur des pratiques et interactions langagières, les « artefacts managériaux nommés « projet » et « qualité » [qui] sont aussi des pratiques communicationnelles », étudiés par Françoise Bernard (2005), sont des puissants révélateurs des demandes du moment. 
- La multiplication de nouveaux outils de traitement de l'information et de mesure d'une activité professionnelle, qui concerne spécifiquement les travaux en communication organisationnelle, notamment dans leurs dimensions stratégiques et d'applications aux processus opérationnels.

Sur ce dernier point tout particulièrement, on sait à quel point les équipements technologiques issus de l'univers informatique et réticulaire exercent une fascination par leur fonctionnalité de saisie, de stockage, de visualisation - dans des configurations et des représentations diverses - des multiples facettes d'une praxis (Meyer, 2005). En définitive, les objets techniques et les logiciels deviennent les pièces maitresses de l'expertise, parce qu'ils permettent d'emmagasiner et d'articuler, "un savoir-faire, c'est-à-dire un répertoire de clés d'action; un savoir-comprendre, c'est-à-dire un moyen d'apprécier jusqu'à quel point il faut mobiliser les savoir-faire ; un savoir-combiner, c'est-à-dire une stratégie pour disposer les différentes phases de son intervention » (Trépos, 1996; 2002 : 8).L'exemple du développement d'une mode managériale comme le Knowledge Management est significatif de cette tendance. Elle montre, s'il le fallait encore, que l'information est définitivement devenue une matière première; ressource essentielle des échanges humains et non-humains, surtout dans la communication au travail. Cette information à l'interne dans une entreprise est surtout constituée des connaissances ordinaires et expertes (intelligence collective), autrement dit des savoirs et savoir-faire qu'il faut aujourd'hui stocker, capitaliser, expertiser et partager. Par cette démarche, on accorde une attention particulière aux temporalités des acteurs et aux relations entre savoir formalisé et expertise, et à celles entre savoirs prescrits/savoirs inventés au quotidien/ savoir-faire de médiation; sans oublier les formes d'élaboration collective des savoirs, ainsi que leur formalisation au sein de règles de métiers, de techniques. On sait aussi que toute activité organisationnelle (surtout dans un champ professionnel), suppose la mise en œuvre de savoirs, très inégalement reconnus il est vrai, quant à leur valeur sociale.

\section{Légitimité des connaissances et statuts des chercheurs}

14 La question du rapport entre l'action et la connaissance croise toujours, à un moment ou un autre, celle de la légitimité de cette dernière, elle-même fortement liée à la question du statut accordé à l'acte du praticien. Dans certains milieux ou secteurs professionnels ( e.g. le tertiaire relationnel, les métiers de service), il est encore peu formalisé et souvent inféodé à d'autres savoirs experts. Bref, les questions s'emboîtent vite: «Qui produit quoi »? Ou, pour reprendre le langage d'autorité classique : « D'où parlent-ils »? Et cette quête de légitimité ne s'arrête que "lorsqu'une activité est constituée en discipline universitaire, [de fait] la question de sa fonction et de la fonction de ceux qui la pratiquent ne se pose plus » (Bourdieu, 1984:47). Les problèmes de qualification des savoirs ne se résolvent pas simplement sous les auspices de l'opératoire qui les rendrait «naturellement» pertinents. Si on peut assez facilement situer et qualifier le discours savant par ses monopoles, en revanche celui issu des RA est généralement suspecté. En premier lieu parce qu'il s'agit d'un savoir spécifié aux besoins des acteurs i.e. des résultats simples immédiatement utilisables sur le terrain. D'aucuns pourraient revendiquer la possibilité de faire exister une praxis sans en référer à une autorité savante. En second lieu, parce qu'il est produit dans une posture de praticien-chercheur qui doit encore et toujours se ressourcer en légitimité. Du reste, très souvent le praticien-chercheur peine - 
peut-être par crainte d'illégitimité - à s'inscrire dans un cadre conceptuel ou construire le sien. Comme le souligne Ruth Canter Kohn (2001: 31): «Opter pour la posture de praticien-chercheur veut dire la coexistence de deux statuts, leurs interactions réciproques constantes, reconnaître qu'une même personne prend plusieurs positions selon les moments, les objectifs, les fonctions, les contacts avec les autres... ». Il faut rappeler ici les tensions (souvent productives pour la réflexion) que génèrent ces positions. Nous nous sommes éloignés quelque peu des interactionnistes du courant de l'école de Chicago, qui avaient accordé de facto une valeur aux positions des savants de l'intérieur.

De plus en plus de professionnels, dans leurs études, ont acquis des rudiments de base de la recherche académique ou reprennent des formations pour les découvrir ou les consolider. Lorsqu'ils décident de s'engager dans une production de connaissances sur leurs pratiques, l'enjeu étant toujours de partir du point de vue des membres pour appuyer des transformations et/ou modernisations, ou répondre à des exigences ou injonctions d'évolution dans leur travail. La RA permet cette confrontation de divers savoirs sur un même objet ou terrain organisationnel, afin de promouvoir cette démarche de rassemblement des connaissances opérationnalisables. Jacques Ardoino (1983: 23) avançait qu'elle "prétend tenir mieux compte de la complexité caractérisant plus spécifiquement les pratiques sociales que tant d'autres approches scientifiques. Susceptible de donner matière à des analyses aussi bien en termes d'effets de force, plus énergétiques, plus organisationnels, qu'en termes d'effets de sens, plus herméneutiques, plus institutionnels, elle se veut, délibérément, avec sa double visée de savoir et de changement, un travail sur les implications ». Ceci est d'autant plus important lorsque, à la base, l'objet et le terrain sont en lien avec une demande (ou commande) sociale; autrement dit, dans l'esprit d'une production de connaissances moins vulgarisée qu'opérationnalisable, que d'aucuns pourraient même qualifier de citoyenne. Dans ce cas, il est vrai que les risques d'instrumentalisation de la recherche sont réels, mais il faut prendre au sérieux cette demande sociale grandissante et développer cette forme spécifique de recueil du savoir qu'est la recherche-action; celle-ci peut aussi être vécue comme un acte politique au sens général du terme.

En l'espèce, la RA permet de contribuer à l'élaboration d'un modèle de décision dans lequel les questions de positionnement des chercheurs se posent de manière assez spécifique. On sait que leur regard dans un milieu professionnel, comme du reste les résultats de leurs investigations, ne sont jamais totalement neutres; l'impact de ces derniers reste cependant très difficile à mesurer. Trop souvent encore présentées comme des expertises endogènes trop peu codifiées ou normées, les RA sont le produit (certes toujours instable) du contenu de multiples expériences professionnelles acquises et peu transmises. Rester à la seule internalisation des résultats de ce type de recherche (ils vont servir pro domo), c'est se priver de données essentielles, à la fois sur les principes pratiques des professionnels, mais aussi sur leur capacité à problématiser leur monde. Le chercheur est souvent surpris des capacités de conceptualisation des agents pris dans l'action, surtout dans une période où ils s'interrogent sur sa justesse ou sur sa valeur. Alfred Schutz (1987: 10) a cette belle définition lorsqu'il explique le système de pertinences des individus impliqués dans une situation (autre que celui du chercheur qui les observe) : «Les faits, les événements et les données ont une structure complètement autre pour le chercheur en sciences sociales. Son champ d'observation: le monde social, n'est pas essentiellement un monde sans structure. Il a une signification particulière et 
une structure pertinente pour les êtres humains qui y vivent, qui y pensent et qui y agissent. Ils ont sérié et interprété à l'avance ce monde par de nombreuses constructions».

\section{Conclusion}

17 Il est toujours nécessaire de rappeler que c'est par l'intermédiaire des démarches méthodologiques, et les techniques de recherche (ou d'enquête) qu'elles requièrent, que se construisent principalement les problématisations et les conceptualisations. Comme le souligne Gilles Ferréol (1995: 73):«La méthodologie regroupe l'ensemble des idées directrices orientant l'investigation empirique. La connaissance est ainsi perçue soit comme un produit qu'il convient de tester ou de valider (logique de la preuve), soit comme un processus dont il importe de reconstituer la genèse (logique de découverte) ». Le problème de la validité des savoirs produits se pose finalement dans tout projet de recherche. $\mathrm{Si}$, avec des outils fiables, la recherche devient validable, les résultats doivent toujours être discutés et le pouvoir explicatif des analyses évalué, notamment dans une restitution des résultats aux groupes ou personnes concernés. En fait, cette démarche n'est qu'une reconstruction humaine, logique et pertinente, temporairement valable et validée, qui se situe en parallèle ou en adéquation du processus réel.

Dans le champ des SIC, la construction et l'usage des données doivent pouvoir être facilement repris sous un angle opératoire. La demande d'un savoir centré et spécifié aux besoins des professionnels existe et grandit. Toutefois ces productions doivent toujours tenir compte de la manière dont les individus s'approprient les résultats et propositions $\mathrm{du}$ chercheur, tout en relativisant leur validité au regard des conditions de production (temporalité, posture et implication du chercheur). Ces précautions étant prises, on peut suivre Brigitte Bouquet et Christine Garcette $(2004: 30)$ pour qui : «Associer la recherche de l'acteur à celle de l'universitaire est riche d'intérêt, car c'est accepter le débat contradictoire, la dialectique nécessaire à la distanciation, c'est conjuguer la logique de proximité de l'acteur à la capacité de distance critique du chercheur ».

\section{BIBLIOGRAPHIE}

Amado G., Guittet A., 1997, Dynamique des communications dans les groupes, Paris, A.Colin.

Ardoino J., 1983, « Conditions et limites de la recherche-action », Pour, 90, pp. 22-26.

Avenier M.-J., 2004, « Transformer l'expérience en savoirs actionnables légitimés en sciences de gestion considérées comme des sciences de conception ", pp. 801-822, in : H. Savall, M. Bonnet, M. Perron, éds, Traversée des frontières entre méthodes de recherche qualitatives et quantitatives, Lyon, ISEOR-IAE de Lyon.

Bernard F., 2005, « Pratiques professionnelles/pratiques communicationnelles : des figures de l'engagement par les actes ", Questions de communication, Série Actes 3. 
Boumard P., 1989, Les savants de l'intérieur - L'analyse de la société scolaire par ses agents, Paris, A. Colin.

Bouquet B., Garcette C., 2004, «Le réseau histoire du travail social », Vie sociale, 2, pp. 27-35.

Bourdieu P., 1980, Le sens pratique, Paris, Éd. de Minuit.

- 1984, Questions de sociologie, Paris, Éd. de Minuit.

Boure R., 2002, «Présentation : Le droit au passé », pp. 9-15, in : R. Boure (éd.), Les origines des sciences de l'information et de la communication, Lille, Presses universitaires du Septentrion.

Canter Kohn R., 2001, « Les positions enchevêtrées du praticien qui devient chercheur », pp. 15-38, in M.-P. Mackiewicz, coord., Praticien et chercheur. Parcours dans le champ social, Paris, Éd. L'Harmattan.

Cristallini V., 2004, « La recherche des invariants en recherche-intervention : justification et méthodes ", pp. 691-704, in : H. Savall, M. Bonnet, M. Perron, éds, Traversée des frontières entre méthodes de recherche qualitatives et quantitatives, Lyon, ISEOR-IAE de Lyon.

Hess R., 1983, « Histoire et typologie de la recherche-action », Pour, 90, pp. 9-16.

Lapassade G., 1991, L'ethnosociologie, Paris, Éd. Méridiens Klincksieck.

Schutz A., 1987, Le chercheur et le quotidien, Paris, Éd. Méridiens Klincksieck.

Trépos J.-Y., 1996, La sociologie de l'expertise, Paris, Presses universitaires de France.

- 2002, "L'expertise comme équipement politique de la société civile », Questions de communication, 2, pp. 7-18.

\section{NOTES}

1. Cette démarche traduit la volonté de : «négocier un cahier des charges de la recherche avec les organisations; de varier les occasions et les modalités de collecte des informations; de varier et rendre hétérogènes les formes d'expression des données, de mener des observations de longue durée dans les organisations » (Cristallini, $2004: 691)$.

\section{RÉSUMÉS}

Une démarche de recherche-action permet de rendre compte de diverses dynamiques et transformations sociales. Plus particulièrement dans les organisations, pareille posture vise à saisir les points de vue des individus et produire avec eux du savoir sur les situations qu'ils rencontrent; en une formule : faire qu'ils deviennent les savants de leur propre réalité. Prenant au sérieux cette possibilité, cet article revient sur l'intérêt des recherches-actions dans le champ des pratiques en information et communication et engage la réflexion sur le statut accordé au savoir « profane » en dehors de sa simple valeur sociale.

A process of research-action enables us to report various dynamics and social changes particularly in organizations. It aims at understanding individuals points of view and producing, 
with them, knowledge on the situations they encounter ; in short, doing what is possible so that they might become knowledgeable in their own reality. Taking seriously this possibility, the article tackles the subject of research-action in the field of practices in information and communication and induces thinking about the statute allowed to "profane" knowledge besides its mere social value.

INDEX

Mots-clés : recherche-action, changement organisationnel, conduite du changement

\section{AUTEUR}

\section{VINCENT MEYER}

Vincent Meyer est Professeur en sciences de l'information et de la communication à l'université Paul Verlaine-Metz. Chercheur au Centre de recherche sur les médiations (EA 3476), ses domaines de recherche sont les pratiques d'information et de communication d'agents individuels ou collectifs dans différents champs professionnels, notamment leur manifestation dans divers discours publics (écrit, oral ou visuel). Ce faisant, il étudie le caractère d'action et d'utilité publique de certaines formes de communication (prévention, dénonciation, médiation, transmission inter-générationnelle, politiques publiques mémorielles, perspectives interculturelle et transfrontalière). Mail : vmeyer@univ-metz.fr 